\title{
Article \\ Evaluation of Patient's Perception and Acceptance of Tele(oral)Medicine for Care during the COVID-19 Pandemic: A Cross-Sectional Pilot Survey
}

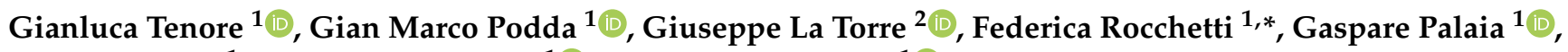 \\ Carlo Di Paolo ${ }^{1}$, Antonella Polimeni ${ }^{1}$ and Umberto Romeo ${ }^{1}$ (D) \\ 1 Department of Oral Sciences and Maxillofacial Surgery, Sapienza University of Rome, 00161 Rome, Italy; \\ gianluca.tenore@uniroma1.it (G.T.); podda.1634638@studenti.uniroma1.it (G.M.P.); \\ gaspare.palaia@uniroma1.it (G.P.); carlo.dipaolo@uniroma1.it (C.D.P.); antonella.polimeni@uniroma1.it (A.P.); \\ umberto.romeo@uniroma1.it (U.R.) \\ 2 Department of Public Health and Infectious Diseases, Sapienza University of Rome, 00161 Rome, Italy; \\ giuseppe.latorre@uniroma1.it \\ * Correspondence: federica.rocchetti@uniroma1.it; Tel.: +39-320-2847201
}

check for

updates

Citation: Tenore, G.; Podda, G.M.; La Torre, G.; Rocchetti, F.; Palaia, G.; Di Paolo, C.; Polimeni, A.; Romeo, U. Evaluation of Patient's Perception and Acceptance of Tele(oral)Medicine for Care during the COVID-19 Pandemic: A Cross-Sectional Pilot Survey. Appl. Sci. 2021, 11, 7443. https://doi.org/10.3390/app11167443

Academic Editor: Andrea Ballini

Received: 16 July 2021

Accepted: 8 August 2021

Published: 13 August 2021

Publisher's Note: MDPI stays neutral with regard to jurisdictional claims in published maps and institutional affiliations.

Copyright: (c) 2021 by the authors. Licensee MDPI, Basel, Switzerland. This article is an open access article distributed under the terms and conditions of the Creative Commons Attribution (CC BY) license (https:/ / creativecommons.org/licenses/by/ $4.0 /)$.

\begin{abstract}
The aim of this cross-sectional pilot survey was to assess patients' perception and acceptance of a tele(oral)medicine program during the COVID-19 pandemic. We conducted a telephone survey on 84 patients of MoMax ambulatory at the Department of Oral Sciences and Maxillofacial Surgery at "Sapienza" University of Rome, Polyclinic Umberto I. Demographic characteristics of the patients were recorded, and a 24 question-survey was completed. A Chi-square test and an ANOVA test were performed with a significance level of $5 \%$ to determine the association between two target questions of the survey (Q22 and Q23) and specific variables such as computer skills, conditions influencing the experience of the conventional visit, and the difficulty in taking intraoral photos. Sixty-two $(73.8 \%)$ patients declared an interest in the use of a tele(oral)medicine program. Furthermore, 41 (48.8\%) participants preferred this type of visit to a conventional odontostomatological visit. Significative statistical associations were found between patients' interest in a tele(oral)medicine service and computer skills, conditions influencing the experience of the conventional visit, and the difficulty in taking intraoral photos. Our study showed that the tele(oral)medicine program appears to meet the expectations and needs of our patients.
\end{abstract}

Keywords: SARS-CoV-2; COVID-19; survey; telemedicine; telehealth; tele(oral)medicine; teledentistry; televisit

\section{Introduction}

The most appropriate definition of telemedicine (TM) is provided by the World Health Organization (WHO), which defines TM as the delivery of healthcare services using information and communications technologies (ICT) for the diagnosis, treatment and prevention of disease as well as research and education in the health field [1]. TM is able to facilitate communication and interaction not only between the healthcare provider and patient but also between the providers themselves. Indeed, it can, to a certain extent, remove geographical and temporal barriers, bridging gaps in the nonhomogeneous distribution of healthcare services [2].

After the declaration made on 11 March 2020 by the WHO, the epidemic of severe acute respiratory syndrome coronavirus-2 (SARS-CoV-2) was proclaimed a global pandemic. The transmission of COVID-19 occurs through liquid particles (respiratory droplets and aerosols) and by contact with contaminated surfaces. A close interaction with an infected patient, distanced less than $1 \mathrm{~m}$, strongly increases the risk of infection [3]. Dentistry is one of the medical branches presenting a high risk of SARS-CoV-2 transmission. The dentist-patient relationship involves face-to-face contact, which is necessary to approach 
the oropharyngeal region. Furthermore, the production of droplets and aerosols and direct contact with the patient's fluids, such as saliva or blood, are characteristic of dental procedures [4]. Following the recent shelter-in-place orders across Italy, the majority of oral medicine clinics in hospitals, dental schools, and private practice settings are faced with the challenge of maintaining regular oral medicine services while keeping their patients, providers, and staff from being exposed to COVID-19 [5].

Following the aim of bridging the gap between healthcare services and the population, tele(oral)medicine has been recognized as a tool to help combat the COVID-19 pandemic.

The following three modes of operation hold great value for oral diagnosis: teleorientation, which allows professionals to perform screenings, provide guidance, and refer patients with suspected oral lesions to face-to-face assistance, if needed; telemonitoring, which permits professionals to visually monitor patients through photographic control; and teleconsultation, which enables the exchange of information between professionals, assisting in diagnosis and therapy for the patient.

Although teledentistry is far from new, it is used on a limited scale and its advantages in tele(oral)medicine have not yet been fully explored. Furthermore, there is limited published evidence to show patients' readiness and acceptance of teledentistry services [6]. The current literature on the acceptance of teledentistry is limited to health care providers [7].

Barriers to the use of telemedicine include concern regarding the patient-physician relationship, legal issues such as privacy and data concerns, and social issues such as variable digital access and familiarity with telecommunications technologies [8].

The aim of this pilot survey was to assess patients' evaluation and acceptance regarding the introduction of a new tele(oral)medicine service to improve patients' satisfaction and, ultimately, patients' care.

\section{Materials and Methods}

All procedures performed in studies involving human participants were in accordance with the ethical standards of the institutional and/or national research committee and with the 1964 Helsinki declaration and its later amendments or comparable ethical standards.

This cross-sectional survey was approved by the local Ethics Committee of Sapienza University of Rome (Ref. 5813, Prot. 0520/2020).

A telephone survey was administered by a single trained interviewer during April 2020. Participants were selected through probabilistic sampling among patients treated at the MoMax (Oral Medicine and Maxillofacial) ambulatory of the Department of Oral Sciences and Maxillofacial Surgery at "Sapienza" University of Rome, Polyclinic Umberto I $[9,10]$. This ambulatory is a task force that was founded in June 2014 and designed to provide cancer patients and patients with Oral Potentially Malignant Lesions (OPML) with multidisciplinary team care. The main aim of this project is to customize and accelerate the treatment plan for cancer patients as a trial to improve their survival rate. Different health providers work together in this project, including oral medicine specialists, prosthodontists, maxillofacial surgeons, oncologists, radiotherapists, and anatomopathologists.

The sample size was calculated using EpiCalc 2000 version 1.02 (Microsoft Windows application) by setting the following parameters:

- Expected answer "Yes" to Question 22 (Would you be interested in using an oral telemedicine service?) $=65 \%$;

- $\quad$ Null hypothesis value $=50 \%$;

- $\quad$ Significance $=0.05$;

- $\quad$ Power $=80 \%$.

The sample size obtained was 84 patients.

The selection of the participants was achieved by generating random numbers using EpiCalc 2000 version 1.02; each generated number was then connected to a specific patient in our department database.

Only patients aged $>18$ years old were included. Patients that met the following criteria were excluded from the study: patients aged $>75$ years old, patients who did not 
answer telephone calls after three tries, patients who did not give consent, patients with language difficulties, and patients with mental disabilities. Each participant was informed about the study, including the following information: interview duration (mean duration: $20 \mathrm{~min}$ ), aim of the survey, guarantee of participant anonymity, and the option to withdraw from the study.

Before the administration of the questionnaire, each participant was required to agree to the consent and privacy policy through audio registration as proof of voluntary participation in the study. The personal data for each patient were anonymized through the assignment of a generic number, and only the researchers were able to identify the patient.

The questionnaire was developed by a panel of four experienced oral pathologists and one biostatician after reviewing the relevant published literature and the most recent available information on telemedicine, teledentistry, and tele(oral)medicine.

The survey consisted of 24 multiple-choice questions divided into four different domains: sociodemographic variables (gender, age, civil status, city of residence, occupation, etc.); factors that may influence the patient's experience within the MoMax ambulatory (annual mean access to our ambulatory, waiting time before the visit, etc.); skills in the use of technology (ability to use information technology (IT) devices, ability to use smartphone cameras, etc.); and interest in tele(oral)medicine services.

The answers were encoded and entered into an Excel spreadsheet (Microsoft Office 2016, Redmond, WA, USA). Statistical analysis was performed in collaboration with the Department of Public Health and Infectious Diseases of the Polyclinic Umberto I, Sapienza University of Rome, using SPSS software version 25.0. Descriptive analysis was reported as frequencies and percentages. Chi-square tests $(\chi 2)$ and one-way analysis of variance (ANOVA) were utilized to determine the associations between the two target questions of the questionnaire, Q22 (would you be interested in using an oral telemedicine service?) and Q23 (how would you define your level of interest in accessing a new oral telemedicine service?), and the following variables: city of residence, profession, gender, age, time taken to get to the hospital from home, personal impact of home-hospital distance, mean annual access to our ambulatory, waiting time before the visit, personal impact of waiting time before the visit, payment exemption for the visit, access to an IT device, ability to use IT devices, need for human contact during the visit, ability to use a smartphone camera, evaluation of complexity of taking an intraoral photo and interest in paying the visit through the web. The statistical significance level was set at $p \leq 0.05$.

\section{Results}

To include 84 patients, it was necessary to select 167 patients through EpiCalc 2000. Eighty-three patients were excluded from the study: 39 patients refused to participate, 44 patients did not answer telephone calls after three times, and one patient was excluded due to language difficulties.

The sex distribution of the patients was 42 (50\%) males and 42 (50\%) females, with a mean age of $53.44 \pm 11.63$ years old. Forty-one $(48.8 \%)$ patients lived in Rome, while $43(51.2 \%)$ patients lived in other cities (Viterbo, Cassino, Terni, etc.). The average number of annual visits to the MoMax Ambulatory was 3.99 \pm 3.60 . The majority of the patients had access to digital devices and possessed enough skill to use these resources. Eighty $(95.2 \%)$ patients claimed to own a computer or a smartphone connected to the internet in their home; forty-six (54.7\%) patients stated that they were skilled in the use of these technologies; of the remaining 38 (45.3\%) patients, 27 stated that they had the possibility of being supported in the use of these technologies by a relative and/or a close friend.

Table 1 shows the frequencies and percentages of answers to our survey. Regarding the complexity of taking an intraoral photograph, $43(51.2 \%)$ patients answered that this procedure was "possible", and 15 (17.9\%) patients answered "easy" (Figure 1). The greatest advantage identified by patients in favor of this technology was the ability to remain at home, while the absence of physical contact was regarded as the greatest disadvantage. For question 22, $62(73.8 \%)$ patients declared that they were interested in the use of a 
tele(oral)medicine service. For question 23, 16 (19.1\%) patients showed a level of interest in a new tele(oral)medicine service defined as "null", while $6(7.1 \%)$ patients had a "low", $37(44.0 \%)$ a "medium", $15(17.9 \%)$ a "high" and $10(11.9 \%)$ a "very high" level of interest. Furthermore, $41(48.8 \%)$ participants declared that they preferred this type of visit to a conventional odontostomatological visit (Figure 2).

Table 1. Frequencies and percentages of answers to our survey.

\begin{tabular}{|c|c|c|c|}
\hline Question & Answer & Frequency & Percentage \\
\hline \multirow{2}{*}{ Q1. Gender } & Male & 42 & 50.0 \\
\hline & Female & 42 & 50.0 \\
\hline \multirow{4}{*}{ Q2. Civil Status } & Single & 20 & 23.8 \\
\hline & Married & 55 & 65.5 \\
\hline & Divorced & 7 & 8.3 \\
\hline & Widower & 2 & 2.4 \\
\hline \multirow{4}{*}{ Q3. Profession } & Employed & 55 & 65.5 \\
\hline & Unemployed & 11 & 13.1 \\
\hline & Student & 3 & 3.6 \\
\hline & Retired & 15 & 17.9 \\
\hline \multirow{2}{*}{ Q4. City of Residence } & Rome & 41 & 48.8 \\
\hline & Other City & 43 & 51.2 \\
\hline \multirow{5}{*}{ Q5. Age } & $21-30$ & 4 & 4.7 \\
\hline & $31-40$ & 11 & 13.1 \\
\hline & $41-50$ & 12 & 14.3 \\
\hline & $51-60$ & 32 & 38.1 \\
\hline & $61-75$ & 25 & 29.8 \\
\hline \multirow{3}{*}{$\begin{array}{l}\text { Q6. Mean annual days accessed } \\
\text { to the ambulatory }\end{array}$} & $1-3$ times & 49 & 58.3 \\
\hline & 4-8 times & 23 & 24.4 \\
\hline & $>9$ times & 12 & 14.3 \\
\hline \multirow{3}{*}{$\begin{array}{l}\text { Q7. Time taken to get to the } \\
\text { hospital from home }\end{array}$} & 00:05 h-01:00 h & 58 & 69.0 \\
\hline & $01: 15 \mathrm{~h}-02: 00 \mathrm{~h}$ & 22 & 26.2 \\
\hline & $>02: 15 \mathrm{~h}$ & 4 & 4.8 \\
\hline \multirow{5}{*}{$\begin{array}{l}\text { Q8. Personal impact of } \\
\text { home-hospital distance }\end{array}$} & Null & 39 & 46.4 \\
\hline & Low & 20 & 23.8 \\
\hline & Medium & 18 & 21.4 \\
\hline & High & 6 & 7.2 \\
\hline & Very high & 1 & 1.2 \\
\hline \multirow{2}{*}{ Q9. Way of reaching the hospital } & Alone & 55 & 65.5 \\
\hline & Accompanied & 29 & 34.5 \\
\hline \multirow{4}{*}{$\begin{array}{l}\text { Q10. Average waiting-time } \\
\text { before the visit }\end{array}$} & 00:05 h-00:30 h & 26 & 31.0 \\
\hline & $00: 30 \mathrm{~h}-01: 00 \mathrm{~h}$ & 17 & 20.2 \\
\hline & $01: 15 \mathrm{~h}-02: 00 \mathrm{~h}$ & 27 & 32.1 \\
\hline & $>02: 15 \mathrm{~h}$ & 14 & 16.7 \\
\hline \multirow{5}{*}{$\begin{array}{l}\text { Q11. Personal impact of } \\
\text { waiting-time before the visit }\end{array}$} & Null & 32 & 38.1 \\
\hline & Low & 19 & 22.6 \\
\hline & Medium & 20 & 23.8 \\
\hline & High & 10 & 11.9 \\
\hline & Very high & 3 & 3.6 \\
\hline \multirow{2}{*}{$\begin{array}{l}\text { Q12. Payment exemption of } \\
\text { medical service }\end{array}$} & Yes & 42 & 50.0 \\
\hline & No & 42 & 50.0 \\
\hline \multirow{2}{*}{ Q13. Access to an IT device } & Yes & 80 & 95.2 \\
\hline & No & 4 & 4.8 \\
\hline \multirow{5}{*}{ Q14. Ability to use IT devices } & Null & 17 & 20.2 \\
\hline & Low & 21 & 25.0 \\
\hline & Medium & 37 & 44.0 \\
\hline & High & 9 & 10.7 \\
\hline & Very high & 0 & 00.0 \\
\hline \multirow{5}{*}{$\begin{array}{l}\text { Q15. Ability to use a smartphone } \\
\text { camera }\end{array}$} & Null & 18 & 21.4 \\
\hline & Low & 17 & 20.2 \\
\hline & Medium & 35 & 41.7 \\
\hline & High & 14 & 16.7 \\
\hline & Very high & 0 & 00.0 \\
\hline
\end{tabular}


Table 1. Cont.

\begin{tabular}{|c|c|c|c|}
\hline Question & Answer & Frequency & Percentage \\
\hline \multirow{5}{*}{$\begin{array}{l}\text { Q16. Reported difficulty in } \\
\text { taking intraoral photos }\end{array}$} & Impossible & 0 & 00.0 \\
\hline & Very hard & 15 & 17.9 \\
\hline & Hard & 11 & 13.1 \\
\hline & Possible & 43 & 51.2 \\
\hline & Easy & 15 & 17.9 \\
\hline \multirow{5}{*}{$\begin{array}{l}\text { Q17. Need for human contact } \\
\text { during the visit }\end{array}$} & Null & 1 & 1.2 \\
\hline & Low & 5 & 6.0 \\
\hline & Medium & 29 & 34.5 \\
\hline & High & 25 & 29.8 \\
\hline & Very high & 24 & 28.6 \\
\hline \multirow{5}{*}{$\begin{array}{l}\text { Q18. Interest in paying the visit } \\
\text { through web }\end{array}$} & Null & 13 & 15.5 \\
\hline & Low & 8 & 9.5 \\
\hline & Medium & 27 & 32.1 \\
\hline & High & 20 & 23.8 \\
\hline & Very high & 16 & 19.0 \\
\hline \multirow{2}{*}{$\begin{array}{l}\text { Q19. Use of other eHealth } \\
\text { platforms }\end{array}$} & Yes & 18 & 21.4 \\
\hline & No & 66 & 78.6 \\
\hline \multirow{5}{*}{$\begin{array}{l}\text { Q20. Major predicted obstacle in } \\
\text { the experience of Telemedicine }\end{array}$} & Use of IT devices & 14 & 16.7 \\
\hline & Internet connection & 5 & 6.0 \\
\hline & Absence of human contact & 35 & 4.7 \\
\hline & Difficulty in taking intraoral photos & 24 & 28.6 \\
\hline & Necessity of paying the visit through web & 6 & 7.1 \\
\hline \multirow{3}{*}{$\begin{array}{l}\text { Q21. Major predicted advantage } \\
\text { in the experience of Telemedicine }\end{array}$} & Lacking need of moving from home & 50 & 59.5 \\
\hline & Lacking need of requesting work-permit & 11 & 13.1 \\
\hline & Visit punctuality & 23 & 27.4 \\
\hline \multirow{3}{*}{$\begin{array}{l}\text { Q22. Interest in accessing a new } \\
\text { Oral Telemedicine service }\end{array}$} & Yes & 62 & 73.8 \\
\hline & No & 15 & 17.9 \\
\hline & Don't Know & 7 & 8.3 \\
\hline \multirow{5}{*}{$\begin{array}{l}\text { Q23. Level of interest in } \\
\text { accessing a new Oral } \\
\text { Telemedicine service }\end{array}$} & Null & 16 & 19.0 \\
\hline & Low & 6 & 7.1 \\
\hline & Medium & 37 & 44.0 \\
\hline & High & 15 & 17.9 \\
\hline & Very high & 10 & 11.9 \\
\hline \multirow{2}{*}{$\begin{array}{l}\text { Q24. Preference towards visit } \\
\text { through e-platform }\end{array}$} & Yes & 41 & 48.8 \\
\hline & No & 43 & 51.2 \\
\hline
\end{tabular}

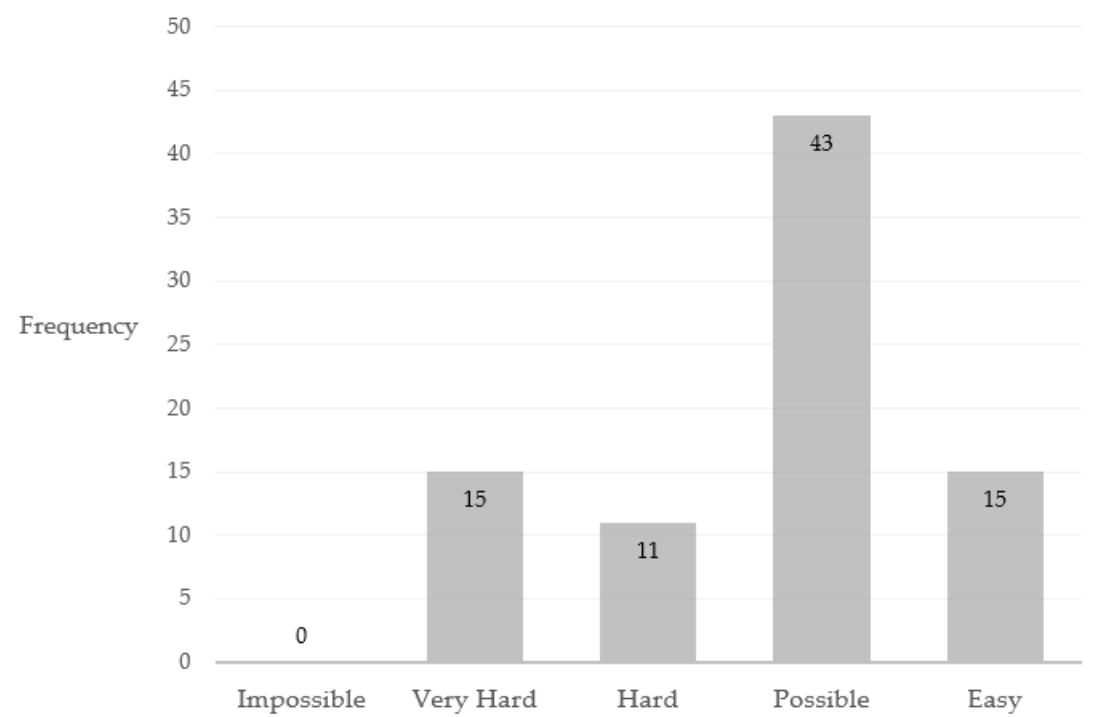

Figure 1. Reported difficulty in taking intraoral photos by study subjects. 


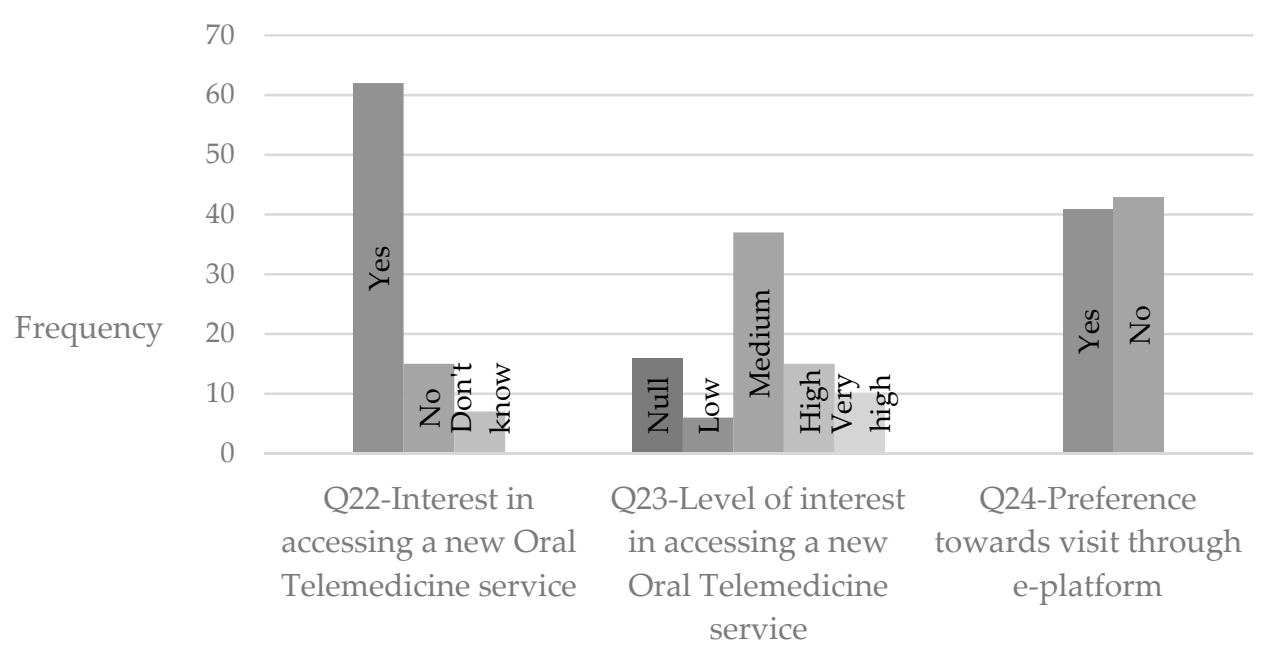

Figure 2. Reported interest in tele(oral) medicine service by study subjects.

Tables 2 and 3 show the results of the bivariate statistical analysis carried out, considering Q22 and Q23 as dependent variables, respectively.

Table 2. Summary of bivariate analysis carried out considering Question 22 as a dependent variable.

\begin{tabular}{|c|c|c|c|}
\hline Variable Related to Q22 & Chi-Square Value & $\mathbf{F}^{1}$ & $p$ Value \\
\hline City of Residence & 0.392 & & 0.531 \\
\hline Profession & 3.364 & & 0.339 \\
\hline Gender & 0.246 & & 0.620 \\
\hline Age & & 3.325 & 0.072 \\
\hline Time taken to get to the hospital from home & 0.189 & & 0.664 \\
\hline Personal impact of home-hospital distance & 8.633 & & 0.071 \\
\hline Mean annual days accessed to the ambulatory & & 1.109 & 0.295 \\
\hline Waiting time before the visit & 8.129 & & 0.017 \\
\hline Personal impact of waiting time before the visit & 4.211 & & 0.378 \\
\hline Payment exemption of medical service & 3.941 & & 0.047 \\
\hline Access to an IT device & 5.176 & & 0.023 \\
\hline Ability to use IT devices & 16.749 & & 0.001 \\
\hline Need for human contact during the visit & 3.286 & & 0.511 \\
\hline Ability to use smartphone camera & 6.779 & & 0.079 \\
\hline Reported difficulty in taking intraoral photos & 11.439 & & 0.010 \\
\hline Patient's interest in paying the visit through web & 41.941 & & 0.000 \\
\hline
\end{tabular}

${ }^{1}$ F-test or Fisher test used in ANOVA analysis.

The $\chi 2$ test demonstrated a statistically significant association between the patients' interest in the tele(oral)medicine service and computer skills (such as the possession of digital devices and the ability to use them); conditions affecting the overall experience of the visit (distance from home, waiting time for the visit, necessity to regularize the binding agreement through web); and difficulty in taking intraoral photos. 
Table 3. Summary of bivariate statistical analysis carried out considering as dependent variables Question 23.

\begin{tabular}{|c|c|c|c|}
\hline Variable Related to Q23. & Chi-Square Value & $\mathbf{F}^{1}$ & $p$ Value \\
\hline City of Residence & 0.134 & & 0.714 \\
\hline Profession & 4.241 & & 0.237 \\
\hline Gender & 0.246 & & 0.620 \\
\hline Age & & 4.788 & 0.032 \\
\hline Time taken to get to the hospital from home & 1.383 & & 0.240 \\
\hline Personal impact of home-hospital distance & 9.982 & & 0.041 \\
\hline Mean annual days accessed to the ambulatory & & 0.967 & 0.328 \\
\hline Waiting time before the visit & 9.020 & & 0.011 \\
\hline Personal impact of waiting time before the visit & 4.036 & & 0.401 \\
\hline Payment exemption of medical service & 3.941 & & 0.047 \\
\hline Access to an IT device & 5.176 & & 0.023 \\
\hline Ability to use IT devices & 9.317 & & 0.025 \\
\hline Need for human contact during the visit & 7.612 & & 0.107 \\
\hline Ability to use smartphone camera & 4.914 & & 0.178 \\
\hline Reported difficulty in taking intraoral photos & 8.788 & & 0.032 \\
\hline Patient's interest in paying the visit through web & 31.097 & & 0.000 \\
\hline
\end{tabular}

${ }^{1}$ F-test or Fisher test used in ANOVA analysis.

\section{Discussion}

Although TM and teledentistry have existed since 1969, in Italy, they are now largely appreciated for their ability to minimize the risk of increased COVID-19 dissemination [11]. With the rapid development of the internet and smartphone apps, telemedicine has transitioned to a multimodal paradigm, offering greater possibilities and convenience.

With the aim of preventing the spread of COVID-19, telemedicine allows for the follow-up of patients who can be remotely monitored, decreasing patient mobilization and reducing the risk of virus dissemination $[12,13]$. The reduction in physical visits and possibility of provider-to-provider communication via the web reduces the risk of infectious exposure for healthcare providers [14]. Despite the high investment that is needed to apply a new TM program, this offers some economic advantages during pandemic emergencies, such as the reduction in costs related to the use of personal protective equipment (PPE) [15] and the option of healthcare providers performing their professional activities remotely when they are asymptomatic and in quarantine [16]. The increase in TM practice in pandemic emergencies is well documented by the Ohio State University Wexner Medical Center, which reported a jump in telemedicine visits from fewer than 100 per day to well over 2200 per day during March 2020 [17] and by NYU Langone Health (NYULH), which recorded a 683\% increase in televisits between March and April 2020 [16].

Regarding oral medicine, there is a complete absence of literature regarding the longterm impact of pandemics on patients with oral potentially malignant disorders (OPMDs) and early-stage oral cancer. Therefore, for healthcare providers who manage OPMDs and oral cancer, a great concern is the risk of progression of these lesions and the harmful sequela in the long term [18]. Although a virtual examination may lack the vital element of palpation, tele(oral)medicine could help to distinguish potentially malignant lesions from those that are truly malignant and require an immediate approach.

To our knowledge, this is one of the first studies that evaluated the use of tele(oral)medicine from a patient's perspective. Our sample included patients who were potential users of a tele(oral)medicine program in the future.

The most interesting result from our survey was that a high percentage of participants $(73.8 \%)$ declared that they were interested in using a new tele(oral)medicine service. This result was also in line with other studies in the specialist literature. Peacock et al. highlighted that $80 \%$ of the patients in their study who were affected by Parkinson's disease would likely use TM for follow-up appointments [19]. Similarly, Valikodath et al. interviewed 97 patients to evaluate their attitude towards TM, and 66 (68\%) participants affirmed 
their willingness to participate in televisits [20]. Abdulhai et al., through interviewing 129 patients at Akron Children's Hospital Paediatric Surgery and Gastrointestinal Clinic, reported that $115(89.2 \%)$ participants were amenable to telemedicine for routine follow-up visits [21].

Furthermore, Villa et al., through evaluating the opinions of 100 patients, emphasized that almost two-thirds of patients thought tele(oral)medicine made it easier to achieve their medical care, and $84.7 \%$ were very satisfied with their telehealth session [22].

Tele(oral)medicine is perceived by patients as a possible means to save time that would otherwise be lost during a medical visit. This explains the statistical association found in our study between the interest in using tele(oral)medicine and the questions investigating the distance of our ambulatory from home (Q8. What is the impact of the home-hospital distance for you? Q23 correlated to Q8 with a $p$ value $=0.041)$, the average waiting time before the visit (Q10. What is the average waiting-time before your visit?; Q22-Q23 both correlated to Q10 with $p$ values of 0.017 and 0.011 respectively) and the possibility of paying the visit through the web (Q18. What is your level of interest in paying the visit through web? $\mathrm{Q} 22-\mathrm{Q} 23$ correlated to Q18 with a $p$ value $=0.000$ in both cases). In our study, the average time taken to reach our ambulatory by the interviewed sample was $65.0 \pm 52.9 \mathrm{~min}$, while the average waiting time before a visit was $81.3 \pm 52.1 \mathrm{~min}$. Telemedicine can significantly decrease travel time and waiting room time compared with in-person visits.

The payment for the visit represents a necessary procedure for the reception of medical practice. The time required for such procedures can be considered as part of the total waiting time the patient undergoes before the visit. The waiting time before the visit constitutes an important factor in the evaluation of the quality of outpatient services [23]. Through the analysis of 11,352 surveys, Bleustein et al. evaluated patients' perceptions regarding waiting time before a scheduled appointment and reported that waiting time is perceived to be not only the main cause of patient dissatisfaction, but also a determinant of care quality [24]. In addition, according to Mohebbifar et al., TM represents a growing opportunity to reduce patient waiting time [25]. Therefore, the opportunity to take payment for the visit through the web and the possibility of performing a digital reception of the patient preceding the visit represents a possible means to further reduce the waiting time before a visit and to achieve a higher quality of care.

The correlation between Q8 and Q23 also illustrates that not needing to move from home is identified as the greatest advantage of Tele(oral)medicine.

The world digitalization will increase the demand for these services by patients. Barr et al. interviewed 1003 participants to evaluate their knowledge about eHealth, stating that $79.4 \%$ of the studied sample had the possibility of accessing the internet [26]. This result is lower than the level of digitalization of our sample: 80 (95.2\%) patients claimed to own a computer or a smartphone connected to the internet. The possession of IT devices and digital skills constitutes a fundamental prerequisite for the use of telemedicine. This explains the statistical association found between these factors and the interest of patients in the use of oral telemedicine systems. Specifically, both Q22 and Q23 showed a statistically significant correlation with Q13 (do you have access to an IT device?), with a $p$ value $=0.023$ in both cases. Furthermore, Q22 and Q23 also showed a correlation with Q14 (what is your level of ability in the use of IT devices?), with $p$ values of 0.001 and 0.025 , respectively.

The need to independently take photographs of the inside of one's oral cavity would constitute a limiting factor for the patients interviewed. Q22 and Q23 showed a statistically significant correlation with Q16 (what is your level of difficulty in taking intraoral photos?) with $p$ values of 0.010 and 0.032 , respectively. This would suggest that patients who perceived taking intraoral photographs to be hard or very hard are less inclined towards such technologies. Therefore, the creation of tutorial videos, or illustration of how to independently take photographs inside the oral cavity, could increase accessibility to these types of services.

Considering that physical touch is key to the relational, physical, and psychological well-being in adults [27], the absence of physical contact could be considered as one of 
the main limiting factors in applying telemedicine. In fact, the absence of physical contact was regarded as the greatest disadvantage of telemedicine by our sample. Nonetheless, no statistically significant association was seen between Q22-Q23 and Q17 (what is your level of need for human contact during the visit?).

The main limitation of this study was that it took place during a period where access to oral healthcare was restricted due to the State of Emergency.

\section{Conclusions}

The COVID-19 pandemic caused great disruption to the dental community and is shaping how we will deliver care in the future. Our study showed that the tele(oral)medicine service appears to be promising among our patients, offering a chance to meet the expectations and needs of our patients.

After the COVID-19 crisis is over, oral medicine specialists may want to consider incorporating TM into their routine clinical practices to benefit patients who must travel long distances or those who must rely on family members or transportation to attend in-person clinical visits.

Author Contributions: G.T.: Conceptualization, Methodology, Validation, Investigation, Data curation, Writing-Original Draft, Writing - Review \& Editing, Supervision, Project Administration; G.M.P.: Conceptualization, Methodology, Investigation, Data curation, Writing-Original Draft, Writing-Review \& Editing; G.L.T.: Conceptualization, Methodology, Validation, Formal analysis, Writing-Review \& Editing; F.R.: Conceptualization, Methodology, Investigation, Data curation, Writing — Original Draft, Writing — Review \& Editing; G.P.: Conceptualization, Methodology, Writing—Review \& Editing; Supervision; C.D.P.: Conceptualization, Methodology, Writing—Review \& Editing; Supervision; A.P.: Conceptualization, Data curation, Supervision, Project Administration; U.R.: Conceptualization, Methodology, Validation, Investigation, Data curation, Writing-Original Draft, Writing - Review \& Editing, Supervision, Project Administration. All authors have read and agreed to the published version of the manuscript.

Funding: This research received no external funding.

Institutional Review Board Statement: The study was conducted according to the guidelines of the Declaration of Helsinki and approved by the Ethics Committee of Sapienza University of Rome (Ref. 5813, Prot. 0520/2020).

Informed Consent Statement: Informed consent was obtained from all subjects involved in the study. Before the administration of the questionnaire, each participant had to agree to the consent and privacy policy through audio registration as proof of voluntary participation in the study.

Conflicts of Interest: The authors declare no conflict of interest.

\section{References}

1. WHO Group Consultation on Health Telematics. A Health Telematics Policy in Support of WHO's Health-for-All Strategy for Global Health Development: Report of the WHO Group Consultation on Health Telematics, 11-16 December, Geneva, 1997; World Health Organization: Geneva, Switzerland, 1998.

2. Maspero, C.; Abate, A.; Cavagnetto, D.; El Morsi, M.; Fama, A.; Farronato, M. Available technologies, applications and benefits of teleorthodontics. A literature review and possible applications during the COVID-19 pandemic. JCM 2020, 9, 1891. [CrossRef]

3. Chu, D.K.; Akl, E.A.; Duda, S.; Solo, K.; Yaacoub, S.; Schünemann, H.J. Physical distancing, face masks, and eye protection to prevent person-to-person transmission of SARS-CoV-2 and COVID-19: A systematic review and meta-analysis. Lancet 2020, 395, 1973-1987. [CrossRef]

4. Peng, X.; Xu, X.; Li, Y.; Cheng, L.; Zhou, X.; Ren, B. Transmission routes of 2019-nCoV and controls in dental practice. Int. J. Oral Sci. 2020, 12, 9. [CrossRef]

5. Villa, A.; Sankar, V.; Shiboski, C. Tele(oral)medicine: A new approach during the COVID-19 crisis. Oral Dis. 2020. [CrossRef]

6. Daniel, S.J.; Wu, L.; Kumar, S. Teledentistry: A systematic review of clinical outcomes, utilization and costs. J. Dent. Hyg. 2013, 87, 345-352.

7. Estai, M.; Kruger, E.; Tennant, M. Perceptions of Australian dental practitioners about using telemedicine in dental practice. Br. Dent. J. 2016, 220, 25-29. [CrossRef]

8. Estai, M.; Kruger, E.; Tennant, M.; Bunt, S.; Kanagasingam, Y. Challenges in the uptake of telemedicine in dentistry. Rural Remote Health 2016, 16, 3915. 
9. De Felice, F.; de Vincentiis, M.; Valentini, V.; Musio, D.; Mezi, S.; Lo Mele, L.; Della Monaca, M.; D'Aguanno, V.; Terenzi, V.; Di Brino, M.; et al. Management of salivary gland malignant tumor: The Policlinico Umberto I, "Sapienza” University of Rome Head and Neck Unit clinical recommendations. Crit. Rev. Oncol. Hematol. 2017, 120, 93-97. [CrossRef]

10. De Felice, F.; Valentini, V.; de Vincentiis, M.; Catalano, C.; Musio, D.; Mezi, S.; Messineo, D.; Di Gioia, C.R.T.; Tenore, G.; Priore, P.; et al. Phase 2 of coronavirus disease (COVID-19) and head and neck cancer: An action plan. Oral Dis. 2020. [CrossRef]

11. Arora, P.; Kaur, J.; Kaur, J.; Arora, A. Teledentistry: An innovative tool for the underserved population. Digit. Med. 2019, 5, 6. [CrossRef]

12. Doshi, A.; Platt, Y.; Dressen, J.R.; Matthews, B.K.; Siy, J.C. Keep calm and log on: Telemedicine for COVID-19 pandemic response. J. Hosp. Med. 2020, 15, 301-304. [CrossRef] [PubMed]

13. Bokolo, A.J. Exploring the adoption of telemedicine and virtual software for care of outpatients during and after COVID-19 pandemic. Ir. J. Med. Sci. 2021, 1-10. [CrossRef]

14. Vidal-Alaball, J.; Acosta-Roja, R.; Pastor Hernández, N.; Sanchez Luque, U.; Morrison, D.; Narejos Pérez, S.; Perez-Llano, J.; Salvador Vèrges, A.; López Seguí, F. Telemedicine in the face of the COVID-19 pandemic. Atención Primaria 2020, 52, 418-422. [CrossRef] [PubMed]

15. Chauhan, V.; Galwankar, S.; Arquilla, B.; Garg, M.; Somma, S.D.; El-Menyar, A.; Krishnan, V.; Gerber, J.; Holland, R.; Stawicki, S.P. Novel coronavirus (COVID-19): Leveraging telemedicine to optimize care while minimizing exposures and viral transmission. J. Emerg. Trauma Shock 2020, 13, 20-24. [CrossRef] [PubMed]

16. Mann, D.M.; Chen, J.; Chunara, R.; Testa, P.A.; Nov, O. COVID-19 transforms health care through telemedicine: Evidence from the field. J. Am. Med Inform. Assoc. 2020, 27, 1132-1135. [CrossRef]

17. Contreras, C.M.; Metzger, G.A.; Beane, J.D.; Dedhia, P.H.; Ejaz, A.; Pawlik, T.M. Telemedicine: Patient-provider clinical engagement during the COVID-19 pandemic and beyond. J. Gastrointest. Surg. 2020, 24, 1692-1697. [CrossRef] [PubMed]

18. Shanti, R.M.; Stoopler, E.T.; Weinstein, G.S.; Newman, J.G.; Cannady, S.B.; Rajasekaran, K.; Tanaka, T.I.; O'Malley, B.W.; Le, A.D.; Sollecito, T.P. Considerations in the evaluation and management of oral potentially malignant disorders during the COVID-19 pandemic. Head Neck 2020, 42, 1497-1502. [CrossRef]

19. Peacock, D.; Baumeister, P.; Monaghan, A.; Siever, J.; Yoneda, J.; Wile, D. Perception of healthcare access and utility of telehealth among Parkinson's disease patients. Can. J. Neurol. Sci. 2020, 47, 700-704. [CrossRef]

20. Valikodath, N.G.; Leveque, T.K.; Wang, S.Y.; Lee, P.P.; Newman-Casey, P.A.; Hansen, S.O.; Woodward, M.A. Patient attitudes toward telemedicine for diabetic retinopathy. Telemed e-Health 2017, 23, 205-212. [CrossRef]

21. Abdulhai, S.; Glenn, I.C.; McNinch, N.L.; Craner, D.; Chou, E.; Ponsky, T.A. Public perception of telemedicine and surgical telementoring in the pediatric population: Results of a parental survey. J. Laparoendosc. Adv. Surg. Tech. 2018, 28, 215-217. [CrossRef]

22. Villa, A.; Sankar, V.; Shazib, M.A.; Ramos, D.; Veluppillai, P.; Wu, A.; Shiboski, C. Patient and providers' satisfaction with tele(oral)medicine during the COVID-19 pandemic. Oral Dis. 2020. [CrossRef]

23. Sibbel, R.; Urban, C. Agent-Based Modeling and Simulation for Hospital Management. In Cooperative Agents; Saam, N.J., Schmidt, B., Eds.; Springer: Dordrecht, The Netherlands, 2001; pp. 183-202. ISBN 978-90-481-5902-4.

24. Bleustein, C.; Rothschild, D.B.; Valen, A.; Valatis, E.; Schweitzer, L.; Jones, R. Wait times, patient satisfaction scores, and the perception of care. Am. J. Manag. Care 2014, 20, 393-400.

25. Mohebbifar, R.; Hasanpoor, E.; Mohseni, M.; Sokhanvar, M.; Khosravizadeh, O.; Mousavi Isfahani, H. Outpatient waiting time in health services and teaching hospitals: A case study in Iran. GJHS 2013, 6, 172. [CrossRef]

26. Barr, P.J.; Brady, S.C.; Hughes, C.M.; McElnay, J.C. Public knowledge and perceptions of connected health. J. Eval. Clin. Pract. 2014, 20, 246-254. [CrossRef]

27. Field, T. Touch for socioemotional and physical well-being: A review. Dev. Rev. 2010, 30, 367-383. [CrossRef] 